\title{
APRENDIZAJE ORGANIZACIONAL PARA LA CONSTRUCCIÓN DE NUEVAS REALIDADES
}

Mgter. Castro Chans, Norma Beatriz ${ }^{(1)(2)}$; Esp. Guastavino Mosna, María Lorena ${ }^{(1)}$, Esp. Goñalons, Gabriela B. ${ }^{(1)}$ y Cavalieri, Ana Belén ${ }^{(3)}$

\section{RESUMEN}

El presente trabajo se propone recuperar la experiencia realizada en el marco de un proyecto de voluntariado universitario entre integrantes de la Universidad Nacional del Nordeste y miembros el Teatro de la Ciudad (Corrientes), sala de teatro autogestionada. La mirada se centra especialmente en los procesos de aprendizaje organizacional que se promovieron a partir de las líneas de acción desarrolladas con el proyecto.

El proyecto de extensión se planteó sistematizar a través de talleres participativos, el trayecto recorrido por la organización, las alianzas y vinculaciones establecidas, las estrategias desplegadas, los logros y las dificultades; y, a partir de allí, establecer y consensuar las líneas de acción para el fortalecimiento institucional. Ambas fases, diagnóstico y planificación, fueron abordados desde el paradigma de la gestión del conocimiento con la intención de que estas acciones se conformen en instancias de aprendizaje y proyección organizacional.
Palabras clave: gestión del conocimiento - teatro - comunicación - extensión - tecnologías

\section{INTRODUCCIÓN}

En las organizaciones culturales el activo más importante y en muchos casos el único lo representan las personas vinculadas a la organización, en la mayoría de los casos son arte y parte. Son personas con alto compromiso y voluntad de trabajo, pero que usualmente no están especializados en cuestiones de administración y gestión organizacional, formación y experiencia que van adquiriendo a partir de las necesidades planteadas por la propia organización. A esto se suman los tiempos propios de las actividades artísticas, la gestión de recursos, las presentaciones de proyectos que dejan poco margen para la planificación y la evaluación organizacional, para recuperar la experiencia y sistematizar la memoria de la organización y valorar el recorrido. En este sentido, entendemos a las manifestaciones artísticas y teatrales como expresiones de la

\footnotetext{
(1) Departamento de Informática. Facultad de Ciencias Exactas y Naturales y Agrimensura (UNNE), 9 de Julio 1449, 3400, Corrientes, Corrientes, Argentina - (ml.guastavino@hotmail.com - gabygonialons@gmail.com)

(2) Dpto. comunicación - Facultad de Humanidades - (UNNE) Sargento Cabral 2001, 3400, Corrientes, Argentina (beatriz.castrochans@comunidad.unne.edu.ar)

(3) Facultad de Artes, Diseño y Ciencias de la Cultura (UNNE) Arturo Illia N 368, 3500, Resistencia, Chaco, Argentina. (anabelencav@hotmail.com.ar)
} 
cultura viva de nuestro pueblo que forma parte del patrimonio cultural intangible. El patrimonio cultural (material o inmaterial) pertenece y es producto de la sociedad, tanto en su materialización original como en su contextualización actual. Pero no siempre la sociedad es consciente del valor que posee ni del alcance del reconocimiento de todo lo que definimos como patrimonio cultural intangible, ni del derecho de las personas al acceso a diversidad de manifestaciones culturales que los identifiquen, los expresen o los hagan sentir parte de una sociedad. Consideramos que con este proyecto hemos aportado a la comunicación, entendida esta en su sentido más antiguo, como diálogo, como intercambio simbólico entre diferentes, para comprender el mundo $\mathrm{y}$ transformarlo.

\section{EXTENSIÓN UNIVERSITARIA COMO ESPACIO DE COMUNICA- CIÓN}

La universidad argentina y algunas latinoamericanas suman una nueva función a las ya instaladas de docencia e investigación a partir de la Reforma de 1918, abriendo el mundo académico a la sociedad. En este sentido, Tamaño y Eciolaza (2008) reconocen que esta variedad de significados se visualiza en las diferentes funciones que se les asignan a las actividades de extensión universitaria, tales como: divulgación de saberes que interesan a la comunidad en su conjunto, apoyo al desarrollo económico y tecnológico ya sea de ciencias básicas como aplicadas, interpelación al poder que descuida temas como el medio ambiente o el desarrollo social, vehículo de las prevenciones en materia de salud y seguridad, o como facilitador de la producción y reproducción de la cultura, entre otros.

Huergo (2004, p.1) siguiendo a Paulo Freire señala que los modelos tradicionales de extensión "trabajan sobre la base de una fuerte distinción entre la cultura de los expertos (los técnicos, los profesionales) y la cultura de los públicos". Esto se traduce en que el propósito de la extensión estaría relacionada con la transmisión de saberes o de informaciones a los sectores a los cuales se considera carentes de saberes. En este significado hegemónico, se hace evidente que la extensión no está centrada en los destinatarios y sus prácticas culturales y productivas, sino en los extensionistas, sus programas y proyectos, sus saberes, las informaciones que tienen para transmitir, sus propósitos y sus formas de actuar.

Entendemos con González González y González Fernández-Larrea (2006) que las características esenciales de la extensión son:

1. La extensión universitaria se produce mediante la actividad y la comunicación,

2. La extensión universitaria se orienta a la comunidad universitaria y a la población en general,

3. La extensión universitaria puede realizarse dentro y fuera de la universidad,

4. La extensión universitaria es parte de las interacciones de la universidad y la sociedad,

5. La extensión universitaria tiene como propósito promover cultura.

La primera representa uno de los rasgos distintivos en relación a los enfoques tradi- 
cionales, ya que permite repensar la extensión como proceso de comunicación en el que la interacción social a partir de la cual se desarrollan las actividades adquiere especial relevancia, oponiéndose de este modo a los enfoques basados en procesos unidireccionales.

Por su parte, Huergo (op.cit) recupera la distinción desarrollada por Paulo Freire entre extensión y comunicación, aludiendo que lo primero hace referencia a donación, entrega, mesianismo, mecanicismo, invasión cultural, manipulación, mientras que la idea de comunicación implica diálogo, intercambio de saberes, articulación cultural, trabajo "con" y no "para" los otros.

En definitiva, la polisemia del término extensión puede ser considerada como uno de los tantos factores que ubica a las actividades de extensión en una posición diferente al de las otras dos funciones centrales de la universidad: la docencia y la investigación, provocando -entre otras consecuencias- la falta de reconocimiento de las actividades de extensión como productoras de conocimiento y solapando, por tanto, la necesidad de ubicar a las mismas dentro del paradigma de la gestión del conocimiento a fin de favorecer y contribuir a la transformación social.

El debate en torno a la cuestión de la extensión universitaria incorpora en la actualidad nuevos ribetes, al respecto, González Fernández-Larrea y González González
(2013) afirman que:

la expansión de saberes (especialización), la diversificación de las fuentes financieras, las nuevas formas de crearse el conocimiento, el creciente carácter práctico de los saberes, el permanente regreso a la aulas con la educación continua, la nueva competitividad por la ex $\neg$ pansión institucional, las nuevas demandas de saberes, la necesidad de construir redes sociales, entre otras, están construyendo ese nuevo contrato y forma de inserción de las universidades en sus sociedades, uno de cuyos componentes más significativos es la pasantía pre-profesional y el voluntariado universitario.

Como anticipamos en la introducción, en esta oportunidad los actores en relación son docentes y estudiantes de la Universidad Nacional del Nordeste e integrantes del Teatro de la Ciudad ${ }^{2}$.

Entendemos a las actividades artísticas y teatrales, como manifestaciones culturales que contribuyen a la construcción de la identidad y se constituyen en motor de desarrollo social. El propósito general de este proyecto es aportar a la valorización de la gestión organizativa y cultural desarrollada por la Asociación Civil Grupo de Teatro "La Trastienda" como responsable de la Sala "Teatro de la Ciudad" en Corrientes. Para lograr este cometido nos hemos propuesto recuperar el trayecto recorrido por la organización, las alianzas establecidas, las estrategias desplegadas, los logros y las dificultades; $y$, a partir de allí, acordar los aspectos centrales que deberían estar inclui-

\footnotetext{
${ }^{2}$ La sala de Teatro de la Ciudad fue inaugurada en noviembre del 2009, y se orientó a constituir un espacio multidisciplinario, donde convergen las artes en sus múltiples manifestaciones, favoreciendo la interacción y el enriquecimiento permanente de cada una de las disciplinas y promoviendo el intercambio de producciones teatrales y otras artes de la provincia, la región y regiones cercanas, brindando al espectador diversas alternativas. También, el Teatro de la Ciudad, a través del Grupo La Trastienda, ha establecido contactos y desarrollado proyectos con el Instituto Nacional del Teatro a través del cual ha logrado, especialmente, mejorar las condiciones edilicias de la sala; también realiza actividades con instituciones educativas de la ciudad de Corrientes, ha participado de instancias, jornadas y foros gestionados por la UNNE. Sin embargo, la que aquí presentamos constituye la primera experiencia de trabajo de gestión conjunta con la universidad que abarca desde la formulación hasta la implementación y evaluación del proyecto.
} 
dos en una planificación estratégica. Ambas fases, diagnóstico y planificación, fueron abordados desde el paradigma de la gestión del conocimiento con la intención de que estas acciones se conformen en instancias de aprendizaje y proyección organizacional. También, el proyecto apunta a fortalecer la imagen del "Teatro de la Ciudad" como organización orientada a la promoción del desarrollo cultural local a través de la elaboración de un plan de comunicación, la producción de soportes comunicacionales y talleres con la intención de captar nuevos públicos, especialmente de sectores que habitualmente están excluidos del acceso a este tipo de manifestaciones culturales. En este sentido, la propuesta no es sólo difundir las actividades de esta Sala, sino aportar información sobre otros espacios y actividades culturales que se ofrecen en la zona (talleres, centros culturales municipales, etc.).

Una organización es consecuencia de la coordinación del trabajo de un grupo de personas; es la búsqueda y conservación del esfuerzo de todos, así como el permanente análisis de los procesos culturales, las necesidades y aspiraciones de la comunidad y de su entorno, que logra hacer de estas aspiraciones y necesidades el objetivo de la organización. La distribución de roles, la toma de decisiones y la gestión de recursos constituyen requisitos necesarios para alcanzar tales objetivos organizacionales. Además, en épocas de cambios tan rápidos como los que están sucediendo es de suma importancia trabajar para que las organizaciones culturales sean flexibles, ágiles y con capacidad de adaptación.

\section{LA COMUNICACIÓN COMO INSTANCIA DE APRENDIZAJE}

Gore y Dunlap (1988, p. 105) definen el aprendizaje organizacional como "el proceso interno de la organización a través del cual se crea conocimiento sobre las relaciones entre las acciones y sus resultados, asi como los efectos del ambiente en la organización." $\mathrm{Y}$ enumeran tres características que debe tener el conocimiento para considerarse organizacionalmente útil: "ser comunicable, ser comprensible para otros miembros de la organización; consensual, considerado válido y útil en forma generalizada; integrado, interrelacionado con otros conocimientos existentes".

En el caso del Teatro de la Ciudad, notamos que la organización se fue complejizando (en términos de la variedad de elementos y aumento de relaciones) para responder a la complejidad y contingencia del entorno. Esta situación significó que unas mismas personas asumieran más de un rol y aumentara la cantidad de tiempo destinado a realizar tareas en la sala. Estas decisiones y la forma de organizarse no se realizaron de manera sistemática ni planificada, sino como respuestas a la coyuntura y en función de las disponibilidades y los intereses de los miembros. Esta forma de organizarse fue encontrando limitaciones para el crecimiento y generando situaciones conflictivas a partir de la sobrecarga de trabajo sobre algunos integrantes, la desmotivación de otros y la aparición de dificultades en el relacionamiento interpersonal.

A partir de esta situación, se realizaron dos talleres participativos para trabajar estos temas. En el primero se recuperó la historia y el proceso organizativo, la participación de diferentes actores a lo largo de los años, los roles asumidos y se puso todo ello en relación con los objetivos iniciales del Teatro de la Ciudad con el objeto de afianzar una visión común e identificar sus logros y dificultades. 
A partir de todo lo producido se fue tomando conciencia de la complejidad de la gestión de la sala y las diferentes dimensiones que requiere sean tenidas en cuenta, se valoró el recorrido realizado y la importancia del aporte y el compromiso de cada uno para alcanzar los objetivos fijados.

En el segundo taller, se partió de los resultados del primero y se trabajó con el método DELIBERA para la selección y jerarquización de problemas, luego se realizó un ejercicio de planificación.

En este proceso el factor central fue la comunicación. Entendemos que "la comunicación- en tanto interacción e interreferenciación social - no solamente construye las relaciones sociales - y los espacios en los que se realizan -, sino que también "construye" a los propios individuos en el proceso de interreferenciación.” (Vizer, 2003, p.247)

Tales procesos surgen en el entramado de la cultura organizacional, entendida como una red de significados expresados a través de símbolos, objetos, rituales que los individuos logran interpretar por el solo hecho de ser parte de esta.

Para este entendimiento es fundamental tomar en cuenta la historia, el contexto, las prácticas sociales y respectivas expresiones. La comunicación no refleja más una realidad, al contrario, esta es 'formativa' en el sentido de crear y representar el proceso de organizar. (Marchiori, 2009).

Entendemos que interacciones comunicativas orientadas al aprendizaje organizacional son los intercambios de sentidos que: tienden a facilitar el diálogo y la construcción de nuevas ideas o conocimiento; incorporan diversas miradas y perspectivas sobre

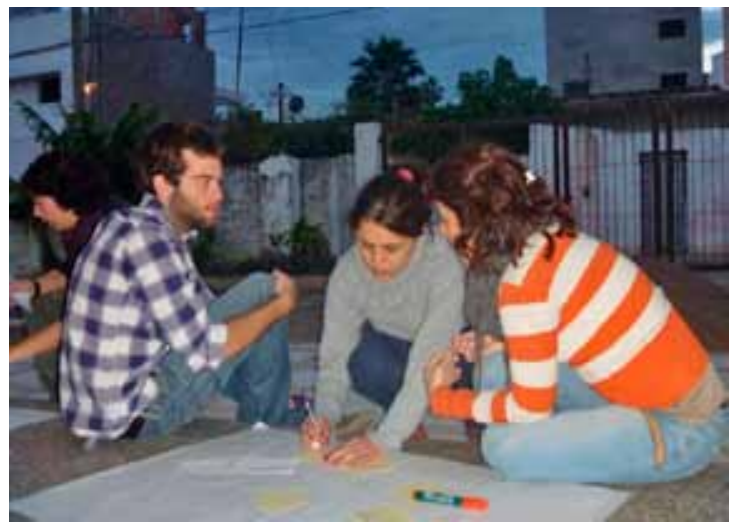

un tema u objeto de estudio; tienen como marco o encuadre una visión común de la organización; los interlocutores consideran que están en condiciones de aportar su experiencia a la organización y que esta se interesa por ello; cuentan con un sistema de información al servicio de todos los actores y de una cultura que asegure la capacidad colectiva para adecuarse a los cambios de la realidad; pueden, o no, estar planificadas (más o menos formalizadas); interactúan con el medio o entorno; y, establecen como prioridad a las salidas del sistema (metas), pudiendo llegar a ellas por múltiples caminos.

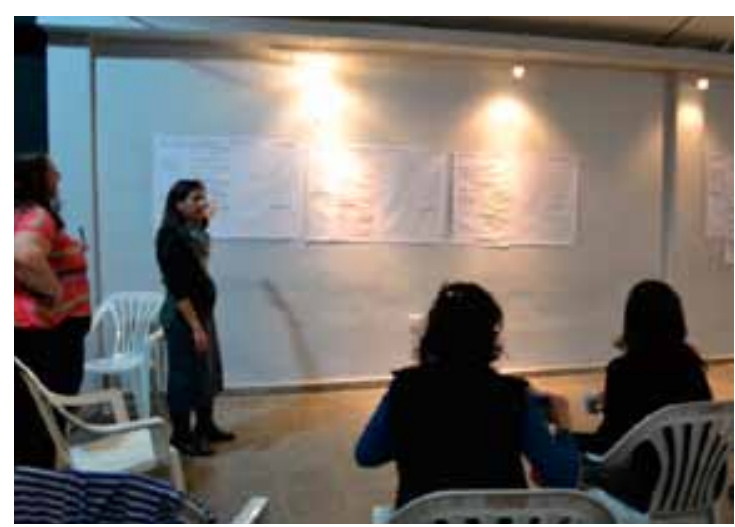

La comunicación interna en una organización implica la interacción de individualidades diferentes comprometidas con la consecución de unos objetivos compartidos, las cuales están ligadas por el desempeño de tareas y por un orden de relaciones que debe ser conocido por todos. Este tipo de interac- 
ciones contribuyen a la construcción de una visión común de la organización y la definición de estrategias propias para alcanzarla, requisitos de las organizaciones inteligentes (Senge, 2005; Pérez Lindo, 2005) y para la gestión del conocimiento organizacional.

Así expuestos, estos ejes presentan una visión de la comunicación interna orientada a la consecución de objetivos comunes de la organización y carente de conflictos y pujas. Sin embargo, así como las interacciones comunicativas operan a favor, también pueden hacerlo contraviniendo los fines o el proyecto organizacional, a través del surgimiento de contraculturas, los conflictos de intereses o de poder, la consolidación de subculturas, el uso de las normas como elemento de presión, entre otros.

Del Moral, A. et.al (2008, p. 32-37) afirman que la gestión del conocimiento en las instituciones debe considerar tres dimensiones: a) El personal y la cultura, en tanto el factor humano constituye un aspecto fundamental en los procesos de gestión del conocimiento. b) La gestión institucional que debe partir de la comprensión de que el conocimiento es un recurso ilimitado, que no se pierde al ser consumido y se revaloriza con su uso. Y, además, que la gestión del conocimiento implica, al menos, tres instancias: "saber lo que se sabe" (conocimientos disponibles para el desarrollo eficiente de su actividad); "saber lo que no se sabe" (conocimientos precisos para garantizar la competitividad de una organización, en el presente $\mathrm{y}$, sobre todo, en el futuro); $\mathrm{y}$ "lo que es necesario saber" (diferencia entre lo que se sabe y lo que se necesita saber, en esta se basará la implementación de técnicas y procedimientos para subsanar esta carencia).c) La tecnología: como herramienta necesaria para una eficaz y eficiente gestión del conocimiento que debe ir en concordancia con la dirección, la visión y la cultura institucional.

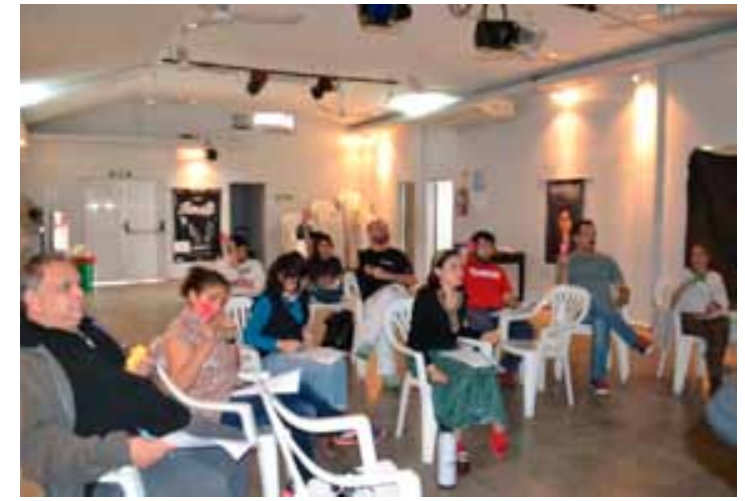

Con relación al uso de TIC, desde hace varios años el grupo que coordina las acciones de la Sala gestiona una página de Facebook donde publican la agenda de actividades e imágenes de las mismas. Además, para la comunicación interna entre los miembros de la organización, utilizan whatsapp y teléfonos móviles. Sin embargo, algo que no habían logrado concretar fue contar con una página web que les permitiera contar con información organizada y disponible para los diferentes públicos. A partir de esa carencia se trabajó en el desarrollo de un sitio web con dominio propio no sólo para difundir información sino para gestionar la reserva de entradas para los espectáculos. El mismo se encuentra actualmente en estudio para implementar mejoras e incorporar nuevos servicios.

La oportunidad que ofrecen los proyectos de extensión universitaria es justamente la posibilidad de construir con el Otro, con los que usualmente se denominan destinarios pero son (o deberían ser) interlocutores y en cuyas relaciones un aspecto central es el aprendizaje colectivo orientado al desarrollo de autonomía.

APRENDIZAJE ORGANIZACIONAL COMO ESTRATEGIA PARA LA CONSTRUCCIÓN DE NUEVAS REALIDADES

El enfoque de gestión del conocimiento 
demanda de parte de los actores una mirada constante sobre el proceso y apertura y creatividad para construir respuestas innovadoras (no previstas), priorizando la producción colectiva por sobre la sectorial.

El aporte de los proyectos de extensión enfocados a la gestión del conocimiento se orientan más hacia el "aprender a aprender" que hacia la transferencia de procedimientos o técnicas específicas, redundando en nuevos conocimientos para el conjunto de los actores involucrados (organizaciones y universidad).

\section{REFERENCIAS BIBLIOGRÁFICAS:}

DelMoral,A.,Pazos,J.,Rodríguez,E.,Rodríguez-Patón, A. y Suárez, S. (2007). Gestión del conocimiento. Madrid: International Thomson Editores.

Freire,P.(2004) ${ }_{\dot{C}}$ Extensióno comunicación?:La concientizaciónenelmediorural. Buenos Aires:SigloXXI.

González González, Gil R.; González Fernández-Larrea, M. (2006) Extensión Universitaria: una aproximación conceptual desde la perspectiva cubana, en Revista Cubana de Educación Superior XXVI (2), Pp. 69 a 76.

González Fernández-Larrea y González González ¿Extensión universitaria, proyección social o tercera misión? una reflexión necesaria. Revista Congreso Universidad. Vol. II, No. 2, 2013, ISSN: 2306-918X

Gore, E. y Dunlap, D. (1988). Aprendizaje y organización: Una lectura educativa de las teorías de la organización. Buenos Aires: Tesis.

Huergo, Jorge (2004) Desafios de la extensión desde la perspectiva cultural. Boletín Dialoguemos, Año 8. No 14. Buenos Aires: INTA, Pp. 9 a 13.

Marchiori, M. (enero - julio 2009). ¿̨Por qué hoy en dia precisamos cultura organizacional? Una perspectiva de comunicación única en el área posmo
Las diversas acciones mencionadas en esta ponencia, desde el acuerdo sobre el significado de las palabras, la comprensión de la complejidad institucional, la construcción de una visión institucional, la identificación de problemas y alternativas de solución, la elaboración de estrategias de intervención, son en sí mismas prácticas de comunicación que contribuyen a la distribución de información y también del poder, donde concurre el conocimiento implícito para tornarse explícito, donde se socializa el conocimiento individual y en las cuales las tecnologías están al servicio del proyecto institu-

derna. Diálogos de la Comunicación, 78, 1-20.

Pérez Lindo, A. (2005) Dimensiones de la Gestión del Conocimiento. En: Pérez Lindo, A. (comp.) Gestión del conocimiento: Un nuevo enfoque aplicable a las organizaciones y a la universidad (pp. 15-78). Buenos Aires: Editorial Norma.

Piñuel Raigada, J. y Gaitán Moya, J. (1997). Teoría de la Comunicación y gestión de las organizaciones. Madrid: Síntesis.

Piñuel Raigada, J. (Septiembre 2010). La noción de mediación comunicativa para el análisis y el diseño de la comunicación organizacional. Revista de Comunicación y Nuevas Tecnologías: Icono 14, 8(2), 125-132.

Senge, P. (2005). La Quinta Disciplina: el arte y la práctica de la organización abierta al aprendizaje (2da ed.). Buenos Aires: Granica.

Tamaño, Gabriela; Eciolaza, Guillermo (2008) La extensión universitaria en la argentina del bicentenario. Disponible en: http://www.ceues.unt.edu.ar/?p=17.

Vizer, E. (2003). La trama (in)visible de la vida social: comunicación, sentido y realidad. Buenos Aires: La Crujía. 